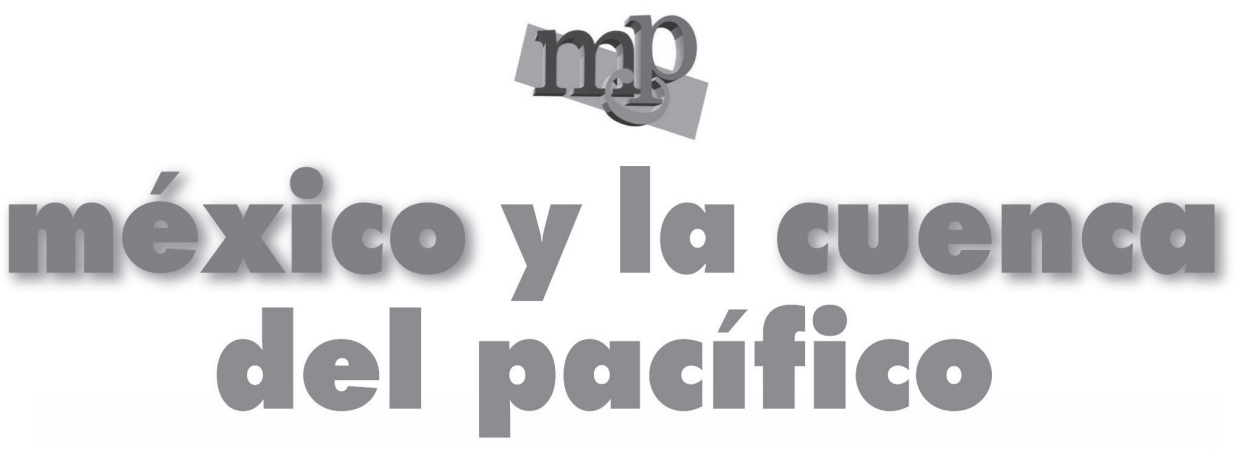

Vol. 12, Núm. 35, mayo-agosto de 2009
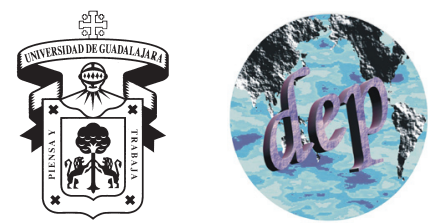

Universidad de Guadalajara

Centro Universitario de Ciencias Sociales y Humanidades Departamento de Estudios del Pacífico 
Mayo - agosto, 2009

D.R. (c) 2009, Universidad de Guadalajara

Departamento de Estudios del Pacífico

Av. de los Maestros y Av. Alcalde, primer piso

ala poniente, edificio G, CP 44260

Guadalajara, Jalisco, México

ISSN: 1665-0174

Impresión: Ediciones de la Noche

UNIVERSIDAD DE GUADALAJARA

Centro Universitario de Ciencias Sociales y Humanidades

Coordinación de Extensión

Guanajuato 1045, Sector Hidalgo

44260, Guadalajara, Jalisco, México

Impreso y hecho en México

Printed and made in Mexico

http://www.publicaciones.cucsh.udg.mx/pperiod/pacifico/index.htm

México y la Cuenca del Pacífico es una publicación cuatrimestral del Departamento de Estudios del Pacífico, del Centro Universitario de Ciencias Sociales y Humanidades de la Universidad de Guadalajara, y tiene como objetivo principal difundir los resultados de investigación de especialistas sobre los aspectos culturales, económicos, políticos y sociales de las economías que integran al Mecanismo de Cooperación Económica Asia-Pacífico (APEC), así como los procesos de integración y de cooperación en la región. Ello con el propósito de contribuir, desde la perspectiva académica, a un conocimiento más profundo de la región de Asia-Pacífico para apoyar la toma de decisiones de los actores involucrados en las relaciones transpacíficas. La revista se encuentra indizada en LATINDEX de la UNAM y en el Catálogo Colectivo de Publicaciones de la Biblioteca Nacional de España. 
UNIVERSIDAD DE GUADALAJARA

Rector general

Dr. Marco Antonio Cortés Guardado

Vicerrector ejecutivo

Dr. Miguel Ángel Navarro Navarro

Secretario general

Lic. José Alfredo Peña Ramos

Centro Universitario de Ciencias

SOCIALES Y HUMANIDADES

Rector

Mtro. Pablo Arredondo Ramírez

Director de la División de Estudios de Estado y Sociedad

Dra. Elisa Cárdenas Ayala

Jefe del Departamento

de Estudios del Pacífico

Dr. Roberto Hernández Hernández

\section{$\mathrm{mp}$ \\ méxico y la cuenca \\ del pacífico}

Directora General

Melba E. Falck Reyes

Director-Editor

José Jaime López Jiménez

Subdirector

Ramón Robledo Padilla

Coordinador editorial

Dagoberto Amparo Tello

Coordinadora de distribución

Nora Preciado Caballero 


\section{$\mathrm{mp}$ \\ méxico y la evencea \\ del pacífico}

\section{Comité editorial}

Melba E. Falck Reyes (Universidad de Guadalajara)

Juan González García (Universidad de Colima)

Roberto Hernández Hernández (Universidad de Guadalajara)

Alfredo Román Zavala (El Colegio de México, A. C.)

Arturo Santa Cruz Díaz Santana (Universidad de Guadalajara)

Carlos Uscanga Prieto (Universidad Nacional Autónoma de México)

Geneviève Marchini (Universidad de Guadalajara)

Omar Martínez Legorreta (El Colegio Mexiquense, A. C.)

Antonio Dueñas Pulido (Universidad del Mar, Oaxaca)

Agustín Jacinto Zavala (El Colegio de Michoacán, A. C.)

Juan José Ramírez Bonilla (El Colegio de México, A. C.)

David Stemper (American University, Estados Unidos)

Román López Villacaña (Universidad de las Américas, Puebla)

Enrique Valencia Lomelí (Universidad de Guadalajara)

Kim Han Sang (Universidad de Kyung Hee, Corea del Sur)

Manfred Mols (Universidad de Mainz, Alemania)

Tani Hiroyuki (Universidad de Sophia, Japón)

José Jaime López Jiménez (Universidad de Guadalajara)

\section{Cuerpo de árbitros}

Yuriko Takahashi (Universidad de Kobe, Japón)

Emilio García Montiel (Universidad Cristóbal Colón de Veracruz, México)

Emma Mendoza Martínez (Universidad de Colima, México)

José Luis León Manriquez (Universidad Autónoma Metropolitana, D. F., México)

Claudia Macías (Universidad Nacional de Seúl, Corea del Sur)

Beatriz Carrillo García (University of Technology, Sydney, Australia)

Catalina Velázquez Morales (Universidad Autónoma de Baja California, México)

Carmen Bueno Castellanos (Universidad Iberoamericana, México)

Abelardo Rodríguez Sumano (UNAM, México)

Jorge Carrillo (Colegio de La Frontera Norte, México)

Cecilia Onaha (Universidad Nacional de La Plata, Argentina).

Víctor López Villafañe (ITESM, México)

Gerardo Traslosheros (Itesm, Campus Ciudad de México)

Carlos Maya Ambía (Universidad Autónoma de Sinaloa)

Jörn Dosch (University of Leeds, UK)

David S. G. Goodman (University of Sydney)

Manfred Wilhelmy (Fundación Asia-Pacífico de Chile)

Alfredo Romero Castilla (UNAM)

Jae Sung Kwak (Kyung Hee University, Korea)

Won-Ho Kim (Hankuk University of Foreing Studies, Korea)

Las opiniones expresadas en los artículos de esta revista son responsabilidad de los autores.

Departamento de Estudios del Pacífico, de la Universidad de Guadalajara,

Av. de los Maestros y Av. Alcalde, Guadalajara, Jalisco.

Tels. y fax 3819-3325 / 3819-3326 e-mail: epacific@fuentes.csh.udg.mx 


\title{
China en el contexto internacional
}

DOI: $10.32870 /$ mycp.v12i35.323

\author{
Roberto Hernández Hernández \\ José Jaime López Jiménez ${ }^{1}$
}

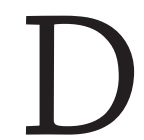

esde finales de los años noventa se ha venido percibiendo a China como una nación en ascenso, conciliadora y propositiva y un miembro cada vez más activo del sistema internacional de la posguerra, diseñado y desarrollado en el contexto de la hegemonía de los Estados Unidos. Dicho en términos del realismo político - expresado por Henry Kissinger-, China pasó de ser una potencia revolucionaria a una potencia defensora del statu quo. Además, China está conociendo cada vez con más detalle los entresijos del sistema internacional y ha sabido aprovecharlos hábilmente en su favor. De esta manera, ha aumentado su habilidad diplomática e incrementado el uso del "soft power"; esto es, la habilidad para influir a otros países mediante la persuasión más que por coerción.

De acuerdo con varios analistas de la política exterior de China, este país ha expandido su influencia en áreas como el sudeste de Asia, África y América Latina, aprovechando el descuido de los Estados Unidos que han puesto mucho más atención - y destinado una gran cantidad de recursos - a la guerra y la lucha contra el terrorismo.

En términos económicos, China ha hecho importantes avances en sus relaciones con el exterior; los datos de comercio e inversión así lo ponen de manifiesto. Lo mismo sucede con su participación e influencia en los principales organismos internacionales. En el proceso de integración del este de Asia el papel de China ha sido determinante, y en regiones como África y América Latina ha abierto mercados para sus productos, así como aprovechado la oferta exportable de materias primas de estas regiones para surtir su planta productiva sedienta de bienes primarios.

1. Profesores-investigadores del Departamento de Estudios del Pacífico, Centro Universitario de Ciencias Sociales y Humanidades, Universidad de Guadalajara. ORCID http:// orcid.org/0000-0002-9145-5865 
Otro aspecto que ha llamado la atención de China en los últimos años son los conflictos entre las minorías nacionales de algunas regiones, y de éstas con los gobiernos regionales y la administración central. Es un hecho que la gran mayoría de los países están integrados por diversos grupos nacionales y que incluso muchos de éstos están en dos o más países, superponiéndose a las fronteras reconocidas por el sistema internacional. Hay que considerar que en tales circunstancias, las lealtades de las personas, familias o grupos son compartidas entre su nación (que les da una identidad cultural) y el país que las incluye, o incluso hacia organizaciones supra-nacionales, como la Unión Europea, que integra varios países. No obstante estas particularidades, el sistema internacional funciona sobre la base de la soberanía del Estado-nación que surgió con el Tratado de Westfalia de 1648.

Para comprender a cabalidad los problemas internos en el Tíbet y Xinjian (y sus particularidades), es necesario analizarlos en el contexto del sistema internacional. Los conflictos entre los grupos nacionales de China, y de éstos con el Estado-nación no son nuevos, ni únicos; con diferentes grados y características, están presentes en la mayoría de los países. Los sucesos que han captado la atención de los espacios mediáticos internacionales, si bien tienen una gran importancia para la estabilidad y el desarrollo interno de China, y trascienden al resto del mundo, no alteran el sistema internacional en su conjunto, fundamentado en la unidad básica del Estado-nación.

En el número anterior de esta revista se abordó el tema del Tíbet. Ahora, dados los conflictos ínter-étnicos de los últimos días (y de julio pasado) en Xinjiang, conviene contextualizarlos para contribuir a su comprensión. En primer término hay que dimensionar el factor demográfico. De acuerdo con el censo de 2000, la población de Xinjiang está integrada por 8.3 millones de uigures (45.2\%), 7.5 millones de chinos Han (40.6\%), 1.2 millones de kazajos (6.7\%), 0.8 millones de hui (4.5\%), y con algunas decenas de miles de habitantes (menos de 1\%) de grupos étnicos como kirguises, mongoles, manchúes, uzbekos, rusos, tártaros y tibetanos, por mencionar los más conocidos.

Otra circunstancia de carácter demográfico es que en general los uigures son la etnia mayoritaria en la parte occidental de Xinjiang, incluyendo las Prefecturas de Kashgar y Khotan, la Prefectura Autónoma Kirguís de Kizilsu y Aksu, así como en la Prefectura de Turfán, en el Xinjiang oriental. Los Han son la etnia mayoritaria en el Xinjiang oriental y septentrional, incluyendo las ciudades de Ürümqi, Karamay, Shihezi y las Prefecturas de Changji, Börtala, Bayin'gholin, Ili (especialmente la ciudad de Kuitun) y Kumul. Los kazajos 
se concentran principalmente en la Prefectura de Ili, en el norte de Xinjiang. Específicamente en Ürümqi (la capital), donde se han presentado los disturbios, la mayoría de la población son Han (75.3\%), uigures (12.8\%), kazajos $(2.3 \%)$ y de otras etnias $(9.6 \%){ }^{2}$

El problema de los disturbios sociales y el separatismo en Xinjiang ha sido una prioridad dentro en la agenda de la seguridad interna de China. De acuerdo con Roy Allison y Lena Jonson, ${ }^{3}$ varios factores explican la situación actual de Xinjiang. Ante todo, hay que considerar el aspecto religioso: desde los años setenta, después de la Revolución Cultural en China, el Islam ha aumentado significativamente el número de creyentes; no sólo entre los uigures, sino también de entre las demás etnias, incluyendo los chinos Han (Xinjiang posee el mayor número de mezquitas en el mundo: un promedio de una por cada 150 habitantes).

En una segunda etapa, desde la segunda mitad de los años ochenta el islamismo radical se ha venido oponiendo a los principios del gobierno de la separación entre el Estado y la religión, específicamente en aspectos como el sistema judicial, el matrimonio y la educación. Los musulmanes radicales han abierto escuelas para el estudio de la religión y han incitado a sus creyentes a participar en una jihad (guerra santa) y a participar en actos terroristas; asimismo, han formado alianzas con otros grupos separatistas.

El separatismo en Xinjiang es otro problema para las autoridades chinas. China siempre ha considerado a Xinjiang como parte inseparable de su territorio. La lucha contra el separatismo tiene una larga historia. Las ideas de pan-islamismo y pan-turkismo surgen desde principios de los años treinta. Conceptos separatistas como la República del Turkestán Oriental han estado presentes desde entonces y el Partido Revolucionario Nacional del Turkestán Oriental ha resurgido en los años noventa, estimulado por los sucesos políticos en Asia central, después de la desintegración de la Unión Soviética que llevó a la formación de estados independientes.

La política del gobierno chino ha sido luchar contra el separatismo y perseguir a los grupos de saboteadores y terroristas sin considerar a la etnia uigur como un todo. No considera el separatismo como un asunto étnico, dada la composición demográfica de Xinjiang, más bien reacciona contra los

2. Disponible en: http://es.wikipedia.org/wiki/Xinjiang. Fecha de consulta: 05/09/09

3. Central Asian Security: The New International Context, Brokings Institution, Washington, DC, 2001, pp. 161-163. 
sentimientos étnicos extremos, estimulados por ciertos sectores del Islam que podrían convertirse en una fuerza social resistente a cualquier control.

Contrariamente a la opinión de algunos sectores interesados en la secesión de estas regiones, los chinos Han no son unos intrusos en el Tíbet ni en Xinjiang. Estas regiones forman parte de la soberanía china, plenamente reconocida por el sistema internacional westfaliano, mismo que da sustento al derecho internacional y las relaciones entre las naciones. Si bien ambas regiones en algún momento de su historia llegaron a albergar pueblos autónomos e incluso llegaron a ser parte activa de amplias conquistas militares (mongolas, por ejemplo), es también un hecho que en otra parte de su historia formaron parte de China.

En un esfuerzo por luchar contra el separatismo y favorecer la estabilidad a largo plazo, el gobierno chino ha puesto énfasis en el desarrollo económico. En este sentido, los gobiernos locales han llevado a cabo políticas para expandir el comercio exterior y estimular la inversión extranjera en proyectos conjuntos con los empresarios de las regiones. Simultáneamente, en un esfuerzo por integrar a estas regiones al desarrollo nacional y ligarlas a la dinámica de la globalización, el gobierno central ha invertido en la construcción de carretas, ferrocarriles, refinerías, canales de irrigación, escuelas y centros de educación superior.

Paradójicamente, el progreso de los chinos Han, reconocidos como laboriosos, ahorrativos y sensatos, ha originado resentimientos entre las comunidades tradicionales que con más dificultad se van integrando a la modernización de China. De este modo, las contradicciones propias del desarrollo, presentes en toda sociedad capitalista o en transición (como se le llama en China), tales como riqueza, pobreza, exclusión y selección laboral en función de habilidades, hicieron brotar resentimientos culturales (lengua, religión, tradiciones, perspectivas tradicionalistas, etc.) entre etnias diferentes y se convirtieron en caldo de cultivo para la acción de grupos extremistas, como los sucesos que el mundo ha presenciado recientemente en territorio chino.

En esta ocasión, la revista incluye cuatro artículos en su sección de Análisis. En su artículo sobre las zonas económicas especiales de China (SEZ, por sus siglas en ingles), Athziri Moreno analiza la relevancia de ese primer experimento chino con la economía de mercado para impulsar la apertura de la economía. La autora hace un recuento, a partir de 1979, de la evolución que las SEZ han experimentado para ajustarse a la dinámica interna del proceso de apertura y al cambiante entorno internacional de las últimas décadas. El mayor reto 
que actualmente enfrentan las SEZ es hacer coincidir el estatus especial que ha privilegiado su funcionamiento, para atraer inversión y fomentar el comercio, con los principios básicos de la OMC de Nación Más Favorecida, Trato Nacional y Transparencia. La autora destaca, además, la influencia del modelo de las SEZ para que otras áreas que no gozaban de los mismos incentivos reclamaran al gobierno central un trato similar. La consecuencia ha sido que la frontera que separaba a las SEZ del resto de la economía se haya ido desvaneciendo y que el proceso de apertura haya permeado hacia otras zonas no incluidas en el experimento original.

En su artículo sobre la migración de mujeres emprendedoras desde China continental hacia la Isla de Hainan, al sur del país, Minglu Chen presenta algunos de los resultados del estudio de campo (2004-2005) que realizó sobre un grupo de mujeres empresarias, en el distrito de Qiongshan de la ciudad de Haikou de la citada provincia. Sobre la base de los lugares de procedencia de las entrevistadas, así como del periodo y el motivo de su migración, la autora encuentra que la tendencia general de la migración ha variado con respecto a las fluctuaciones de la economía isleña así como a las restricciones legales al movimiento poblacional impuestas por el gobierno chino. Es así como la mayor migración se da en el periodo de auge económico de la isla a fines de los años ochenta, cuando el gobierno central considera a Hainan como el lugar ideal para emprender el experimento de una zona económica especial que pudiera convertirse en la ventana que mostrara el nuevo modelo de reforma. Por su estructura productiva y social, la isla constituía la versión en miniatura del país en su conjunto. El desarrollo de la industria de la construcción, del sector de bienes raíces, del sector de turismo y de la agricultura de hortalizas ha marcado la evolución económica de la isla y a su vez ha constituido un fuerte atractivo para la migración. Las mujeres entrevistadas por Minglu Chen vieron en ello una oportunidad de superación. Sin duda, este tipo de estudios micro-regionales arroja luz sobre los procesos migratorios internos en la China pos-reforma.

Por su parte, David S. G. Goodman, en su trabajo sobre las clases medias en China, aborda el fenómeno de la creciente desigualdad regional en términos del ingreso entre la población china, como resultado del rápido crecimiento experimentado por su economía, lo que la ha llevado a convertirse en una de las sociedades con los mayores índices de desigualdad del mundo. Para el autor, desde finales de los años ochenta y principios de los noventa, los principales beneficiarios y agentes de este importante crecimiento económico han sido una gran variedad de nuevos empresarios quienes a escala grande y pequeña han 
sido señalados cada vez con mayor frecuencia, dentro y fuera de China, como la nueva "clase media" o "clases medias". En este sentido, la pregunta central del trabajo gira en torno a si estos nuevos empresarios ricos se pueden identificar como el equivalente de las clases medias de contextos socioeconómicos anteriores. El autor expone que, si bien en algunas provincias de China se ha desarrollado cierto comportamiento de clase media, en especial en cuanto a patrones de consumo, es necesario tener precaución sobre lo mucho que se dice con respecto a los procesos de cambio social en China, y muy particularmente acerca del surgimiento de una nueva clase media en el gigante asiático, en la que se incluye por igual a los chinos sumamente ricos y extra-ricos.

Por último, Marcos Pablo Moloeznik contribuye al debate teórico y analítico sobre el fortalecimiento del poder naval de la Armada de México, con la finalidad de preservar sus derechos y riquezas en las zonas contigua y económica exclusiva, así como de enfrentar a la delincuencia organizada en aguas marinas. El autor sostiene que a pesar de la extensa superficie marina, en la que México ejerce libre soberanía para explorar, explotar, conservar y administrar los recursos vivos y no vivos de los fondos marinos, incluidos los del subsuelo y del agua subyacente, las fuerzas navales mexicanas no guardan correspondencia por su pobre poder naval, al considerarse a la marina de guerra mexicana como costera, modesta y anticuada. En otras palabras, la Armada de México carece de las capacidades mínimas que requiere una marina de guerra, a la cual el soberano le asigna un conjunto de misiones en tiempos de paz y de guerra, mismas que se sintetizan en el ejercicio efectivo de la soberanía en los espacios marítimos. Para el autor, la naturaleza del poder naval mexicano se puede definir como de policía marítima, guardacostas o prefectura, cuyos medios sólo le permiten un control real sobre el mar territorial y parcial sobre la zona contigua.

Las otras cuatro secciones permanentes de la revista sobre Noticias de la Cuenca del Pacífico, a cargo de Ana Bertha Cuevas; Ecos del Pacífico en México, a cargo de Cecilia Escobedo; Nuevas adquisiciones bibliográficas del DEP, bajo la dirección de Diana Serrano y Actividades Académicas del DEP, coordinada por Gabriela Palacios, cierran este número.

Agradecemos a todos nuestros colaboradores el apoyo brindado a México y la Cuenca del Pacífico, a los miembros del Comité Editorial y del Cuerpo de Árbitros por su generosa labor de arbitraje para sostener la calidad de la revista y al Centro Universitario de Ciencias Sociales y Humanidades de la Universidad de Guadalajara por el apoyo para la publicación de la misma. mi 